\title{
Damage detection in structural systems by improved sensitivity of modal strain energy and Tikhonov regularization method
}

\author{
Alireza Entezami • Hashem Shariatmadar
}

Received: 27 September 2013 / Revised: 18 February 2014 / Accepted: 21 February 2014 / Published online: 11 March 2014

(C) Springer-Verlag Berlin Heidelberg 2014

\begin{abstract}
In this article, new methods for detecting damage in structural systems are presented. These methods are categorized as damage localization and damage quantification, respectively. Hence, direct changes of modal strain energy are applied to identify locations of damage. Moreover, some restraints such as incomplete measured modes and simple assumptions in structural modeling may cause failure in the results of damage localization. Therefore, a correlation-based method is utilized to obviate these limitations and precisely detect damage sites. Subsequently, an improved sensitivity of modal strain energy is generated to determine damage severities. To achieve appropriate results in damage quantification, Tikhonov regularization approach is utilized instead of classical methods such as applying penalty function and current inverse problem techniques. Applicability and effectiveness of proposed methods are numerically verified using two practical examples consisting of a planner truss and a portal frame, respectively. Eventually, numerical results indicate that the proposed damage localization approach provides an influential algorithm for precisely identifying damage sites. Furthermore, obtained damage severities show that utilizing the sensitivity of modal strain energy and also solving the damage equation by Tikhonov regularization makes it possible to accurately determine damage extents in the case of incomplete modal data.
\end{abstract}

Keywords Structural damage detection - Modal strain energy - Correlation-based method - Sensitivity analysis . Tikhonov regularization method

A. Entezami $(\bowtie) \cdot H$. Shariatmadar

Department of Civil Engineering, Faculty of Engineering, Ferdowsi

University of Mashhad, Mashhad, Iran

e-mail: entezami@stu-mail.um.ac.ir

H. Shariatmadar

e-mail: shariatmadar@um.ac.ir

\section{Introduction}

Vibration-based damage identification technique has become a popular research topic in the fields of civil, mechanical and aerospace engineering in the last two decades. Based on this technique, structural damages in the form of stiffness reduction would alter the system's vibrational properties i.e. modal frequencies, mode shapes and damping ratios. Hence, many attempts have been carried out to use changes of modal parameters for localizing as well as quantifying structural damages. In this regard, Doebling et al. [1], Stubbs et al. [2] and Salawu [3] and Yan et al. [4] have reviewed previous studies in the literature regarding damage detection process. Moreover, the majority of techniques have detected any type of damages in dynamical systems by direct changes in both physical properties and vibrational responses. Hence, Yan and Golinval [5] presented a damage diagnosis technique based on changes in dynamically measured amounts of flexibility and stiffness in structures. Yang and Sun [6] proposed a new method based on the best achievable flexibility changes in order to localize and quantify damage in dynamical structures. Their damage localization approach consists of computing Euclidean distances between the measured flexibility changes and the best achievable flexibility changes. Furthermore, a damage detection process was carried out by Kim et al. [7] in which changes of both natural frequencies and mode shapes were used. They utilized a methodology to locate and estimate size of damage in structures for which a few natural frequencies or a few mode shapes are available. Esfandiari et al. [8] proposed a novel frequency-based technique to detect any number of localized damages that induce stiffness reduction in a structure. Other methods that are based on changes in the modal parameters are known as modal strain energy methods. In this regard, Yan and Ren [9] applied a statistic structural damage detection algorithm 
developed from closed form of element's modal strain energy sensitivity. Hu et al. [10] detected surface cracks in a circular hollow cylinder using ratio of modal strain energies of the cylinder before and after occurrence of damage. Indeed, they developed a scanning damage index by moving damage indices obtained from local area throughout the structure. Shi et al. [11] used modification of modal strain energy for localizing the damage and then proposed a new method for quantifying damage by means of modal strain energy's sensitivity. Furthermore, Seyedpoor [12] developed a twostage method to identify the damage site and then determine severity of multiple damage cases in structural systems. In the first stage of this method, damage localization approach was improved by a modal strain energy-based index which was previously suggested by Shi and Law [13].

Moreover, sensitivity-based approach is applied in developing damage detection techniques by many researchers. In dynamical systems, sensitivity is defined as the ratio of changing amount of some modal parameters like natural frequencies and mode shapes to the small changes in some of model's properties including mass or stiffness matrices [14]. Accordingly, Li et al. [15] studied various methods for determining sensitivity of element's modal strain energy and then presented a new sensitivity for modal strain energy according to the effects of mass and eigenproblem components. Yan and Ren [16] proposed first-order sensitivity formulas for element of modal strain energy in a real symmetric undamped system based on the algebraic eigensensitivity method. Wang et al. [17] presented a feasibility damage detection method using structure's modal strain energy as a parameter employed in correlation-based method for truss bridge structures. They obtained sensitivity of modal strain energy from the analytical model in which this sensitivity matrix was incorporated into the correlation objective function. It is worth mentioning that detecting damage by means of modal strain energy's sensitivity is not simple due to complexities of the energybased method. On the other hand, finite difference method is utilized in most damage detection approaches to determine the first derivative of structures' physical properties in sensitivity analysis. Hence, the obtained results include errors caused using approximated mathematical equations. Thus, researchers have focused on developing equations that can overcome the aforementioned problems and reducing errors in damage detection. One of the most appropriate methods for obviating the vagueness is to use the minimizing techniques. Consequently, this study aims at eliminating these weaknesses by means of an improved sensitivity for modal strain energy and an efficient minimizing equation.

In this article, improved vibrational-based techniques are used to detect damages in the structural systems. This process is divided to two stages that are categorized as damage localization and damage quantification, respectively. In the first stage, direct alteration of modal strain energy is developed to identify damage sites. In the damage localization approach, some issues can effect detection of damage locations. Thus, a correlation of modal strain energy is introduced to overcome limitations like measurement of incomplete modes and simplifier assumptions in structural modeling. In the second stage, damage severities are determined by an improved sensitivity of modal strain energy. To achieve appropriate results in damage quantification, Tikhonov regularization approach is utilized rather than classical methods like penalty function and current inverse problem techniques. In fact, Tikhonov regularization is an inverse problem which can deal with some ill-conditioned and complicated solutions of other inversing techniques. The applicability and effectiveness of proposed methods have numerically been verified using two practical examples consisting of a planner truss and a portal frame. Eventually, numerical results indicate that the proposed damage localization approach provides an influential algorithm for precise identification of damage sites. Moreover, obtained damage severities show that utilizing the sensitivity of modal strain energy and solution of damage equation by Tikhonov regularization leads to accurate determination of damage extents.

\section{Theory}

\subsection{Modal strain energy}

Free vibration equations for a linear undamped discrete system of $n$ degrees of freedom can be given by

$K \varphi_{i}=\lambda_{i} M \varphi_{i}, \quad i=1,2, \ldots, n$

where $M$ and $K$ are mass and stiffness matrices, respectively. Furthermore, $\lambda_{i}$ and $\varphi_{i}$ are the $i$ th eigenvalue (square of natural frequency, $\lambda_{i}=\omega_{i}^{2}$ ) and eigenvector (mode shape), respectively. Moreover, $n$ is total number of structure's degrees of freedom. To attain appropriate results of modal strain energy, mode shapes should be normalized with respect to mass matrix. Generally, mass matrix is a nonsingular matrix; thus normalization of the $i$ th mode shape can be expressed as:

$\varphi_{i}^{T} M \varphi_{i}=1$

Since mode shape vectors are equivalent to nodal displacements of a vibrating structure, strain energy is stored in each element of the structure [12]. The strain energy of a structure due to mode shape vector are usually referred to as modal strain energy (MSE) and can be considered as a valuable parameter for damage identification. Hence, global modal strain energy for the $i$ th mode of the structure can be defined as:

$M S E=\frac{1}{2} \varphi_{i}^{T} K \varphi_{i}$ 
It is assumed that global stiffness matrix $K$ is assembled by $j$ individual element stiffness matrices $k_{j}$. Hence, if ne is the total number of elements in the structural system, the $j$ th element's MSE for the $i$ th mode can be given by:

$M S E_{i j}=\frac{1}{2} \sum_{j=1}^{n e} \varphi_{i}^{T} k_{j} \varphi_{i}$

\subsection{Damage localization by a new modal strain energy method}

Modal strain energy is determined by combining stiffness of structure's members and mode shapes based on Eqs. (3) or (4). When structural damage occurs, both modal data and physical properties of the structure will be changed. As a result of change in modal data, modal strain energy alters and these changes can be utilized to localize damages. Accordingly, a new damage localization approach is proposed using changes of MSE in the structural system. As Eq. (4) does not provide comprehensive and complete information regarding damage sites, normalized form of MSE is used. This normalized form can be expressed as:

$\eta_{M S E}=\sum_{j=1}^{n e}\left|\frac{\varphi_{i}^{T} k_{j} \varphi_{i}}{\sum_{j=1}^{n e} \varphi_{i}^{T} k_{j} \varphi_{i}}\right|$

where $\eta_{M S E}$ represents the normalized modal strain energy of the $j$ th element in the structure's $i$ th mode of vibration. In practice, obtaining all of modal data is usually impossible. Therefore, normalized MSE for only $m$ identified modes can be rewritten as:

$\xi_{M S E}=\frac{1}{m} \sum_{i=1}^{m} \eta_{M S E}$

where $\xi_{M S E}$ is defined as the finite normalized modal strain energy for undamaged structures. Direct changes of the finite normalized MSE can be applied to detect damage locations. In this regard, modal strain energy of damaged structure should be determined by replacing the damaged mode shapes into Eq. (4). It is clear that the damaged normalized modal strain energy is similar to the typical equation in the normalized MSE for the undamaged structure. Hence, finite normalized modal strain energy for damaged structure $\bar{\xi}_{M S E}$ can be expressed as:

$\bar{\xi}_{M S E}=\frac{1}{m} \sum_{i=1}^{m} \bar{\eta}_{M S E}$

where $\bar{\eta}_{M S E}$ similarly defines as the normalized modal strain energy of $j$ th element in the $i$ th modes for damaged structure:

$\bar{\eta}_{M S E}=\sum_{j=1}^{n e}\left|\frac{\bar{\varphi}_{i}^{T} k_{j} \bar{\varphi}_{i}}{\sum_{j=1}^{n e} \bar{\varphi}_{i}^{T} k_{j} \bar{\varphi}_{i}}\right|$
In the above equation, $\bar{\varphi}$ denotes the mode shape of damaged structure. Occurrence of damage causes an alteration in the MSE and consequently the efficient parameter $\eta_{M S E}$ changes for $m$ modes. As a result, modal strain energy index $(\beta)$ is defined to directly identify the location of damages as follows:

$\beta=\frac{\bar{\xi}_{M S E}-\xi_{M S E}}{\xi_{M S E}}$

It should be noted that it is possible to determine damage locations by Eq. (9) and using modal strain energy alterations. However, obtained results of damage localization may fail due to some reasons including simple assumptions made while modeling the structure, existence of both incomplete modes and contaminated modal data. On the other hand, Eq. (9) is a relative indicator that determines the relation of modal strain energy between undamaged and damaged structures. Thus, the mentioned weaknesses in the damage localization process have more effects on results when MSE index is utilized. Therefore, a correlation-based approach is applied to reduce the effect of these limitations. In other words, determining the level of correlation between undamaged and damaged modal strain energy will provide an efficient tool for locating damages. This correlation method is usually called damage localization assurance criterion [18, 19]. Hence, Eq. (9) can be improved by modal strain energy damage localization indicator (MSEDLI) as follows:

$M S E D L I=\left|\frac{\left(\beta^{T} \cdot \Delta \xi_{M S E}\right)^{2}}{\left(\beta^{T} \cdot \beta\right)\left(\Delta \xi_{M S E}^{T} \cdot \Delta \xi_{M S E}\right)}\right|$

where $\Delta \xi_{M S E}$ is discrepancy between the finite normalized modal strain energy in the undamaged and damaged structures that can be described as $\Delta \xi_{M S E}=\bar{\xi}_{M S E}-\xi_{M S E}$. This correlation method utilizes enhanced multipliers to increase the accuracy of damage localization results and reduce any weaknesses in this process. Indeed, Eq. (10) can detect any type of damage cases by using direct changes of modal strain energy. Accordingly, the MSEDLI in the damaged elements is equal or close to one. In contrast, the MSEDLI indicator is equal to zero for undamaged elements. As a result of computational errors, the MSEDLI is not occasionally equal to one or zero for damaged and undamaged elements, respectively. However, the proposed damage localization approach is in most cases able to determine locations of damages precisely.

\subsection{Damage quantification using improved sensitivity of modal strain energy}

Sensitivity analysis describes rates of changes in vibrational response parameters such as natural frequencies and mode shapes with small alterations in some of the physical properties consisting of individual mass and stiffness matrices [14]. Therefore, derivative of structures' dynamic responses with 
respect to physical properties is usually described as sensitivity analysis in dynamical structures. As discussed before, modal strain energy has been defined by mode shapes and stiffness components. Hence, it is possible to introduce a sensitivity of MSE by first-order derivative of Eq. (4) as follows

$$
\frac{\partial M S E_{i j}}{\partial \alpha}=\sum_{j=1}^{n e}\left(\varphi_{i}^{T} k_{j} \frac{\partial \varphi_{i}}{\partial \alpha}+\frac{1}{2} \varphi_{i}^{T} \frac{\partial k_{j}}{\partial \alpha} \varphi_{i}\right)
$$

As sensitivity of mode shape overcomes the singular problem, the most important ambiguousness of Eq. (11) is difficulty in determining the first derivative of mode shape with respect to damage variable $\alpha$ [15]. To deal with this difficulty, an efficient sensitivity modal strain energy is utilized which was previously proposed by Yan and Ren [16]. In fact, they derived a compact analytical expression for sensitivity of element's MSE based on the algebraic method. In this method, sensitivity of modal strain energy is computed as follows:

$$
\frac{\partial M S E_{i}}{\partial \alpha}=\varphi_{i}^{T} K^{*} \varphi_{i}
$$

where

$$
\begin{aligned}
K^{*}= & {\left[\begin{array}{ll}
k_{j} & 0
\end{array}\right] \cdot\left[\begin{array}{ll}
K-\lambda_{i} M & -M \varphi_{i} \\
-\varphi_{i}^{T} M & 0
\end{array}\right]^{-1} \cdot\left[\begin{array}{l}
\lambda_{i} \frac{\partial M}{\partial \alpha}-\frac{\partial K}{\partial \alpha} \\
\frac{1}{2} \varphi_{i}^{T} \frac{\partial M}{\partial \alpha}
\end{array}\right] } \\
& +\frac{1}{2} \frac{\partial k_{j}}{\partial \alpha}
\end{aligned}
$$

As noted above, this method is a straightforward approach for computing the sensitivity of MSE and does not require using derivative of mode shapes. Indeed, this technique has omitted the mode shape sensitivity and has applied direct derivatives of physical properties of undamaged structures. Hence, first derivatives of the mass and stiffness matrices are simply calculated using finite difference method as follows:

$$
\begin{aligned}
& \frac{\partial K}{\partial \alpha}=\frac{K\left(\alpha_{0}+\Delta \alpha\right)-K\left(\alpha_{0}\right)}{\Delta \alpha} \\
& \frac{\partial M}{\partial \alpha}=\frac{M\left(\alpha_{0}+\Delta \alpha\right)-M\left(\alpha_{0}\right)}{\Delta \alpha}
\end{aligned}
$$

This technology has become a very popular method and has been implemented in many common finite element programs. In this study, it is assumed that damage is directly related to a decrease in the stiffness matrix and the effect of changes in mass can be neglected. Therefore, derivative of mass matrix in Eq. (13) is omitted to introduce improved sensitivity of modal strain energy for damage quantification. Neglecting the mass matrix modification, Eq. (13) can be rewritten as:

$K^{*}=\left[\begin{array}{ll}k_{j} & 0\end{array}\right] \cdot\left[\begin{array}{ll}K-\lambda_{i} M-M \varphi_{i} \\ -\varphi_{i}^{T} M & 0\end{array}\right]^{-1} \cdot\left[\begin{array}{l}-\frac{\partial K}{\partial \alpha} \\ 0\end{array}\right]+\frac{1}{2} \frac{\partial k_{j}}{\partial \alpha}$
Once Eq. (16) is generated, the sensitivity of MSE can be determined with respect to Eq. (12). Thus, the improved sensitivity of modal strain energy is presented as:

$$
\begin{aligned}
& S_{M S E}=\varphi_{i}^{T} \\
& \quad \times\left(\left[\begin{array}{ll}
k_{j} & 0
\end{array}\right] \cdot\left[\begin{array}{ll}
K-\lambda_{i} M & -M \varphi_{i} \\
-\varphi_{i}^{T} M & 0
\end{array}\right]^{-1} \cdot\left[\begin{array}{l}
-\frac{\partial K}{\partial \alpha} \\
0
\end{array}\right]+\frac{1}{2} \frac{\partial k_{j}}{\partial \alpha}\right) \varphi_{i}
\end{aligned}
$$

Having determined modal strain energy's sensitivity, different methods can be utilized to compute damage severities. One of these methods is Taylor series which can be defined as follows:

$x_{k+1}=x_{k}+\frac{\partial f}{\partial \alpha} \Delta \alpha+\frac{\partial^{2} f}{\partial \alpha^{2}} \Delta \alpha^{2}+\cdots+\frac{\partial^{k} f}{\partial \alpha^{k}} \Delta \alpha^{k}$

For damage detection process, Taylor series is truncated to produce a unique expression by neglecting higher order terms in Eq. (18) which can be described as:

$x_{k+1}=x_{k}+\frac{\partial f}{\partial \alpha} \Delta \alpha$

Equation (19) can be used in many vibrational methods. Consider $x$ and $f$ are the vibrational response of structure and the function of dynamic behavior, respectively. Therefore, a damage equation is defined as follows:

$\Delta x=\frac{\partial f}{\partial \alpha} \Delta \alpha$

where $\Delta x$ is an error function between the vibrational responses in the $k$ and $k+1$ states. Generally, this equation is known as penalty function (damage equation) that is widely applied in the both system identification and damage detection techniques. On the other hand, Eq. (20) can be written in more detail for dynamical systems as:

$\Delta \varphi=S \cdot \Delta \alpha$

where $\Delta \varphi$ represents the discrepancy between modal parameters of both undamaged and damaged structures. Furthermore, $S$ is the first-order derivative (sensitivity) of dynamical systems components and $\Delta \alpha$ is also defined as unknown quantity which must be determined. In this regard, a currently used minimization method like Least-square is utilized to solve penalty function as follows:

$\min \|S \cdot \Delta \alpha-\Delta \varphi\|$

As mentioned, Eq. (21) defined as damage equation or penalty function. The damage severities are determined by minimizing this equation. Hence, Eq. (22) is traditionally applied to minimize damage equation. With this simple minimization algorithm, damage quantification process may yield an unacceptable result. Indeed, the least square method is an approximate technique due to neglecting higher order terms. 
Moreover, as the equation of penalty function is often illconditioned and established by some assumptions; direct least-squares solution cannot provide appropriate damage severities if modal parameters are incomplete or when are contaminated by noise. To avoid these problems, regularization is introduced. The some most widely used regularization methods are Tikhonov regularization, truncated singular value decomposition (SVD), truncated generalized SVD and so on [20-23]. Among these techniques, Tikhonov regularization method is the most currently used one. Indeed, this method improves the conditions of the linear problem, which enables a direct numerical solution. Therefore, in the present study, Tikhonov method is adopted to redefine the linearized least-squares problem as minimization of Tikhonov objective function as follows:

$$
J(\alpha+\Delta \alpha)=\left\|S_{M S E} \cdot \Delta \alpha-\Delta \varphi\right\|+\gamma\|\Delta \alpha\|
$$

where $\Delta \alpha$ and $\gamma$ are damage severity and regularization parameter, respectively. Furthermore, $S_{M S E}$ is the improved sensitivity of modal strain energy that has been formulated based on Eq. (17) and using undamaged structure's information. In fact, the Tikhonov objective function is formulated by adding an additional norm to the traditional least-square minimization technique. This added norm is known as solution norm that is adjusted by a regularization value. For example, when $\gamma=0$, minimizing Eq. (23) changes to the least squares estimates. For $\gamma=1$, minimizing the Tikhonov objective function yields the Bayesian estimates. For arbitrary $\gamma>0$, minimizing the objective function yields the Tikhonov-regularized technique with the regularization parameter. Hence, the regularized solution of Eq. (23) depends on the regularization parameter $\gamma$, which controls the weight given to the solution norm $\Delta \alpha$ with respect to residual norm $\left\|S_{M S E} . \Delta \alpha-\Delta \varphi\right\|$. This parameter should be estimated in order to obtain meaningful amount for unknown quantities or damage severities when regularization methods are utilized. Accordingly, L-curve method (LCM) and generalized cross validation (GCV) approach are two popular methods to determine the regularization parameter [24-27]. LCM is a $\log -\log$ plot of the solution norm $\|\Delta \alpha\|$ versus the residual norm $\left\|S_{M S E} . \Delta \alpha-\Delta \varphi\right\|$ [28]. Indeed, the curvature of the $\mathrm{L}$-curve is a function of the regularization parameter which is obtained from the first-and-second order derivatives of the residual norm $[29,30]$. The L-curve basically consists of two parts including a flat part where the regularization error dominates and a steep part where the perturbation error dominates. Consequently, the optimal regularization parameter is determined somewhere near the L-curve's corner. In contrast, the generalized cross-validation method offers a way to estimate proper value of the regularization parameter. In fact, GCV is utilized to maximize the predictability of the regularized solution by appropriate setting of the regularization parameter. In this method, the optimal regularization parameter is calculated as the one that attains the minimal average prediction error for all omitted data points [31-33]. Comparing the capability of two methods, it can be concluded that GCV is a very robust method and provides more proper results in comparison with the L-curve approach. Generally, LCM requires the calculation of the first and second derivatives of the residual norm and the solution norm that involves considerable computational amounts with occasional errors in results. Furthermore, this approach is based on an intuitive heuristic and seeks to balance the two error components via inspection (manually or automated) of the L-curve. Hence, in this study; the generalized cross-validation method is utilized to determine the regularization parameter in the each damage state. Having estimated the regularization parameter, damage variable $\Delta \alpha$ is obtained by minimizing the Tikhonov regularization objective function from the following equation:

$\left[\begin{array}{l}S \\ \gamma I\end{array}\right] \Delta \alpha=\left[\begin{array}{l}\Delta \varphi \\ 0\end{array}\right]$

It should be noted that if $\gamma$ is extremely small, then the objective function of Tikhonov regularization will be too close to the original ill-conditioned problem. In addition, if $\gamma$ is extremely large, then the Eq. (23) will greatly deviate from original problem. Consequently, final regularized solution after minimizing the objective function of Tikhonov regularization method can be expressed as:

$\Delta \alpha=\left(S^{T} S+\gamma I\right)^{-1} S^{T} \Delta \varphi$

According to Eq. (25), damage severity can only be determined by calculating sensitivity of modal strain energy in undamaged structure and also difference between modal parameters of the structure before and after occurrence of damage. Furthermore, applying the regularization parameter is a basic difference between the proposed method and traditional linear least square technique. This parameter increases the accuracy of damage quantification results and also decreases computational errors and some weaknesses such as errors in structural modeling, existence of incomplete modes and contaminated modal data in this process. On the other hand, unlike classical methods, it is not necessary to determine sensitivity of damaged structures to obtain damage severities due to utilize Tikhonov regularization method. Furthermore, it can be deduced that the proposed damage quantification approach is potentially able to compute damage severities in the case of incomplete modal data.

\subsection{Noisy measurement}

In experimental modal tests, there may be some deviations in the results due to the existence of noise in measurements. In the numerical examples, this noise is simulated by adding a series of pseudo-random numbers to the theoretically calcu- 
Fig. 1 Planner truss considered to validate the proposed damage detection methods

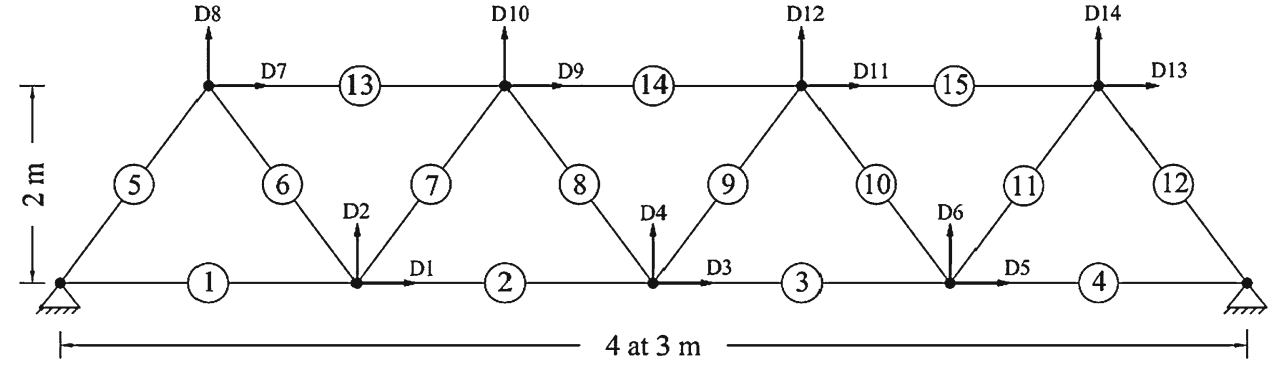

lated frequencies and mode shapes [34]. In other words, due to the complexity of the measurement process, an amount of noise may be inserted in measured data which contaminates the modal parameters. Thus, in order to investigate the effect of noise on the results obtained by proposed damage detection method a random noise is considered as follows:

$\varphi_{i}^{*}=\varphi_{i}\left(1+\theta_{r}\right)$

where $\varphi^{*}$ and $\varphi$ are the eigenvector (mode shape) components of the $i$ th mode with and without noise, respectively. Moreover, $\theta_{r}$ is a random number. In this study, two values equal to 1 and $5 \%$ are applied to mode shapes and natural frequencies as proportional random noises. Accordingly, the damage detection algorithm will be repeated by a series of error-contaminated data created by Eq. (26).

\section{Application}

\subsection{A planner truss}

To illustrate characteristics of the proposed damage detection algorithms, a two-dimensional truss is considered as shown Fig. 1. Basic parameters of the structure's material are Young modulus $\mathrm{E}=200 \mathrm{GPa}$ and density $\rho=7850 \mathrm{~kg} / \mathrm{m}^{3}$. All of truss's members are modeled as L-shaped double equal angles of $100 \mathrm{~mm}$ width and $5 \mathrm{~mm}$ thickness. Each node of the truss has two degrees of freedom. In this example, the first three vibrating modes of the structure are used to detect damages. In the following, two types of random noises including 1 and $5 \%$ are imposed on the both extracted mode shapes and natural frequencies, respectively.

This 2-D truss is a continuous dynamical system and the mass and stiffness matrices can be determined by basic concepts of finite element method [35]. After calculating physical properties of undamaged truss, generalized eigenvalue problem is used to identify modal data. It is assumed that proportional damping dominates structure's behavior and so modal parameters are extracted as real data. Subsequently, four damage cases are considered to investigate effectiveness of proposed methods for damage detection. Accordingly, in the first damage case, stiffness of elements 2 and 14 are reduced by $15 \%$. In damage case number two, the

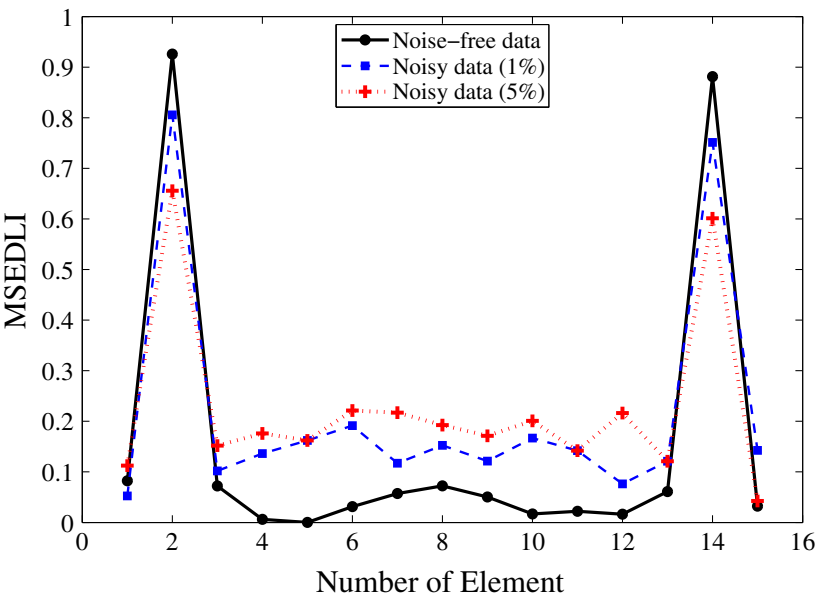

Fig. 2 Damage localization in the planner truss by MSEDLI (damage case 1)

stiffness of elements 6,9 and 12 are decreased by 20,25 and $30 \%$, respectively. In damage case number three, stiffness of elements 3,8 and 15 are reduced by 10,15 and $20 \%$, respectively. Finally, in the fourth damage case, stiffness of element 10 is decreased by $20 \%$. Induced damage cases change the properties of the planner truss and so its vibrational responses. Initially the modal strain energy indices of damaged and undamaged structures are generated based on Eqs. (6) and (7) to localize damages. Next, locations of induced damages are probed by proposed damage localization coefficient index. Therefore, damage locations that were induced in the planner truss can be indicated as follows:

As shown in Figs. 2, 3, 4 and 5 induced damages in the planner truss are exactly identified by the MSEDLI indicator. These figures demonstrate that the proposed method for damage localization is significantly able to locate single and multiple damages cases even in the cases of incomplete modes and the existence of contaminated modal data. Moreover, it can be concluded that results obtained from correlation-based method has preferable performance comparing to calculation of direct changes of the modal strain energy using Eq. (8). Furthermore, Figs. 2, 3, 4 and 5 illustrate the effect of noises in the MSEDLI diagrams. These errors cause some perturbations in the final results of damage localization, particularly in the undamaged elements. Although, computational 


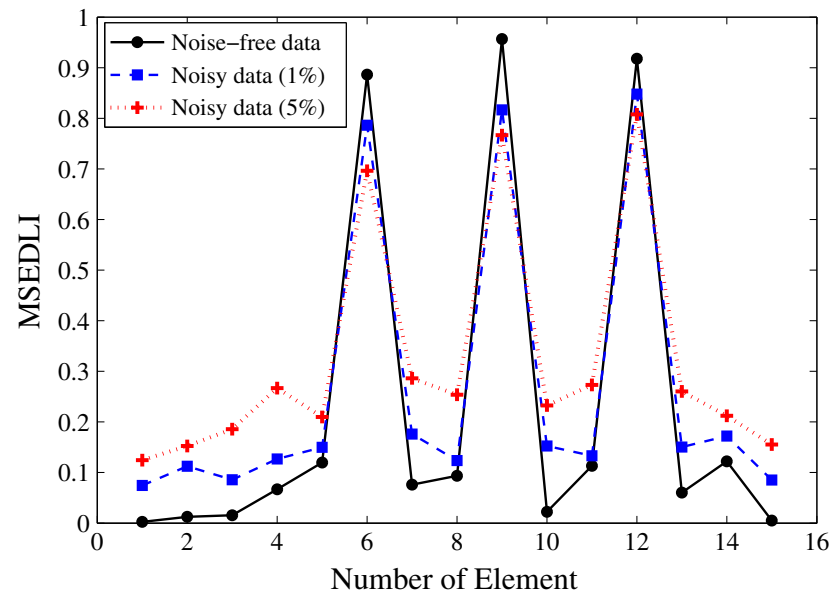

Fig. 3 Damage localization in the planner truss by MSEDLI (damage case 2)

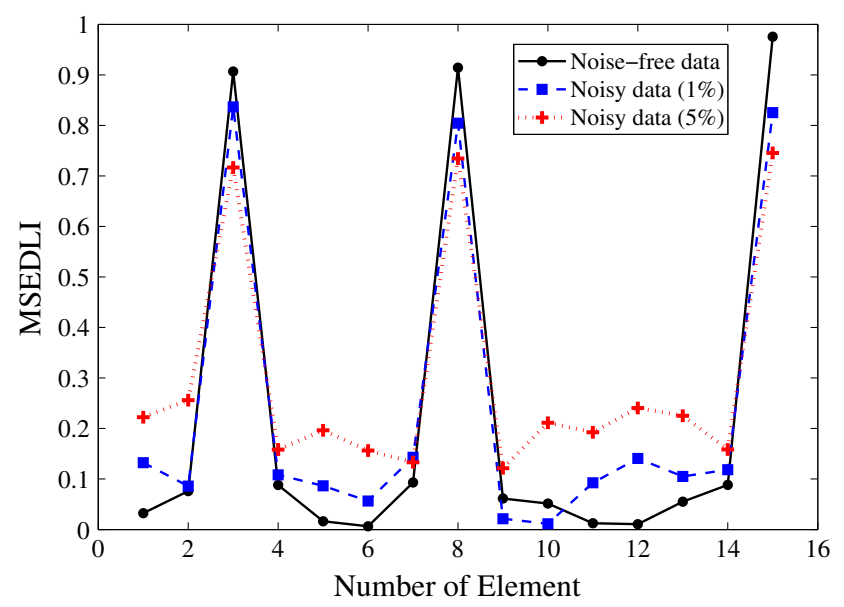

Fig. 4 Damage localization in the planner truss by MSEDLI (damage case 3)

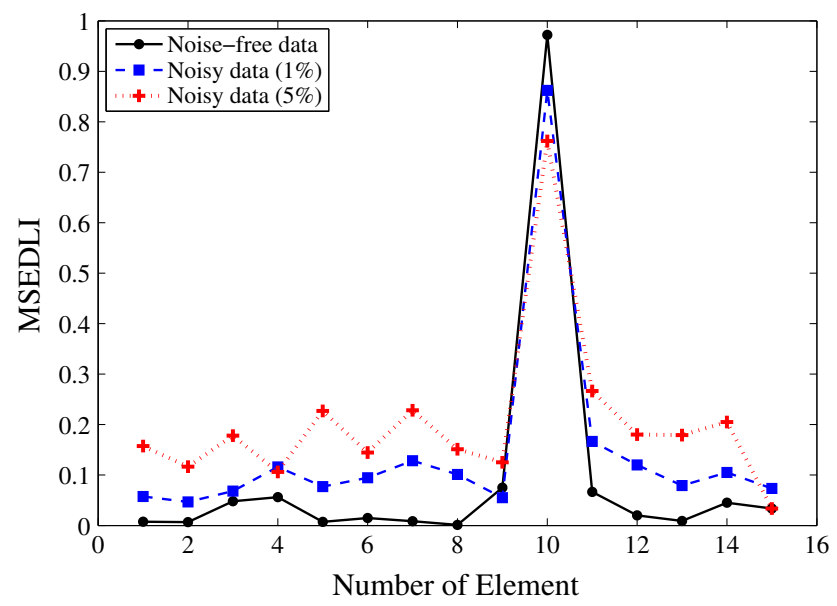

Fig. 5 Damage localization in the planner truss by MSEDLI (damage case 4)

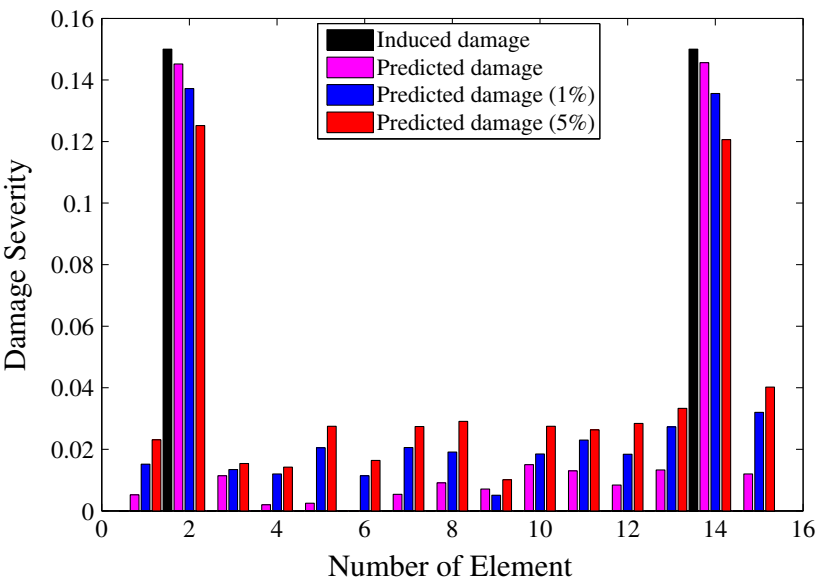

Fig. 6 Damage quantification in the planner truss (damage case 1)

errors in the MSEDLI method have been estimated between 10 and $18 \%$, the locations of damages have been identified precisely. Therefore, it can be concluded that the proposed damage localization approach has high capability to detect any type of damage site when incomplete and contaminated modal data are present.

After precise localization of structural damages, their severities are determined using improved sensitivity of modal strain energy. Next, this sensitivity matrix is utilized in the objective function that is related to Tikhonov regularization technique. In the each damage case, the regularization parameter is calculated by GCV method based on initial information of damage detection process such as sensitivity of physical properties and modal parameters. Final results of damage quantification are calculated using Eq. (25). This process is a complicated mathematical process that requires too many computational efforts for attaining accurate results in damage quantification. Thus, obtained results are confirmed only when the difference between induced and predicated damages is negligible. Figures 6, 7, 8 and 9 illustrate amounts of induced and damage severities in the planner truss as well as three levels of noise effects.

As shown in the above figures, severities of induced damages are calculated with a good precision, even when the noisy data pollute the modal parameters. In other words, obtained numerical results show that in cases of multiple damages and despite incompleteness of measured modes, amount of computational error is less than $5 \%$ in the noisefree condition. On the other hand, this error is estimated between 11 and $18 \%$ when 1 and $5 \%$ random noises contaminate the modal parameters, respectively. Indeed, these errors are acceptable quantities and indicate that the proposed damage quantification method can provide reliable and efficient results when incomplete and contaminated modal parameters are available. Moreover, there are amounts of damage severities predicted in undamaged members of the truss. This may 


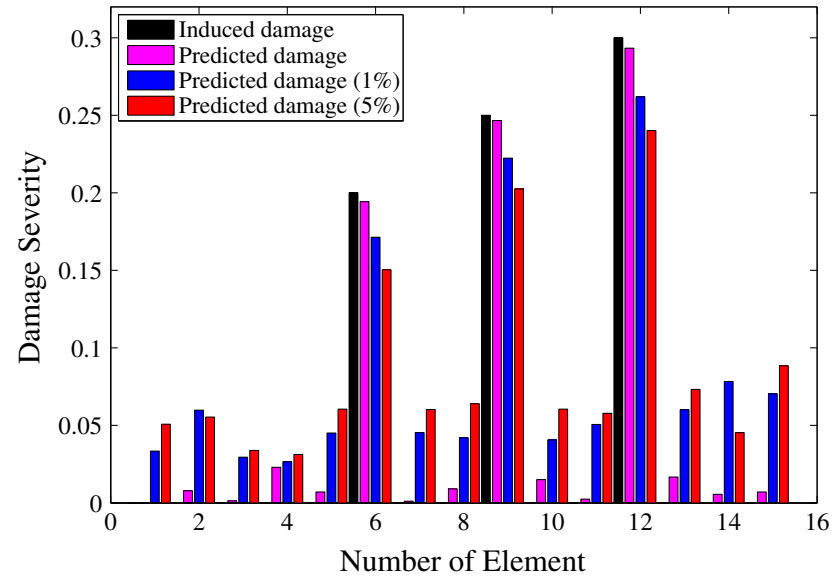

Fig. 7 Damage quantification in the planner truss (damage case 2)

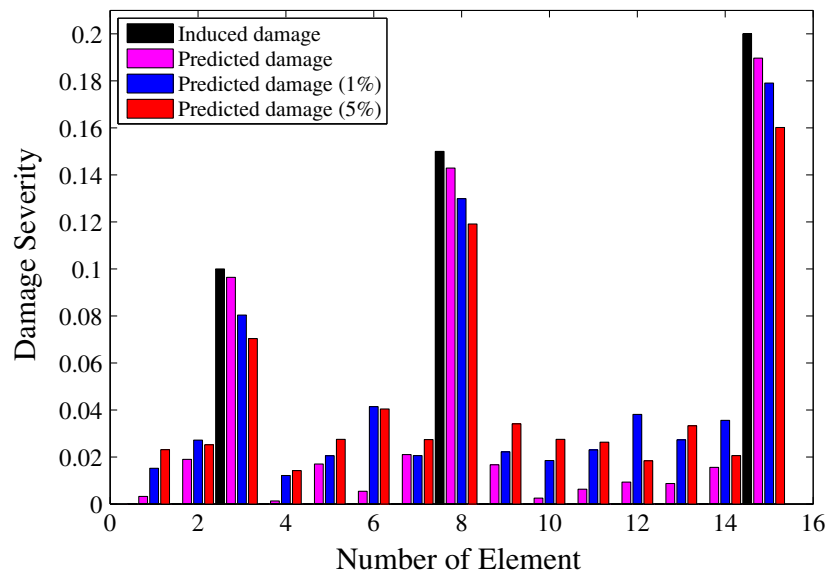

Fig. 8 Damage quantification in the planner truss (damage case 3)

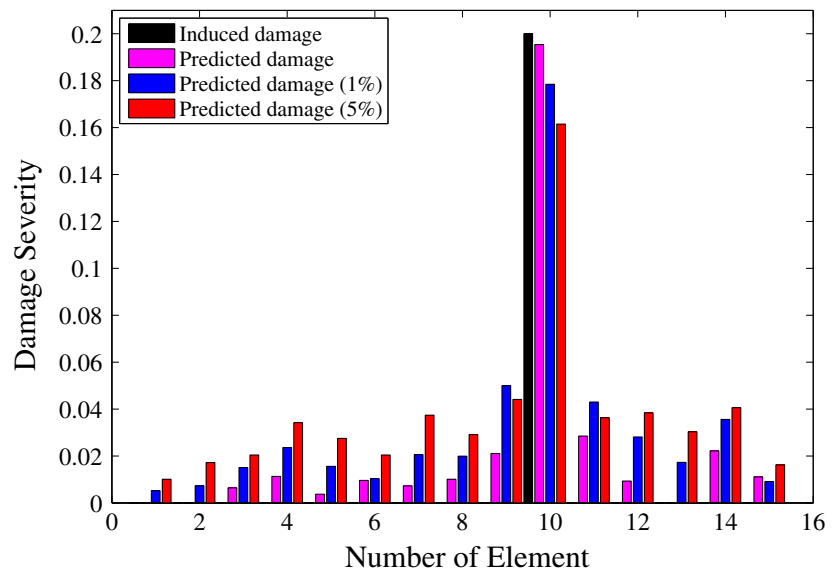

Fig. 9 Damage quantification in the planner truss (damage case 4)

be caused by complexity in calculation of sensitivity matrix, existence of noisy data and also modal data's being incomplete. However, as will be shown later, this error is negligible and does not have any significant effect on damage quantification algorithm.

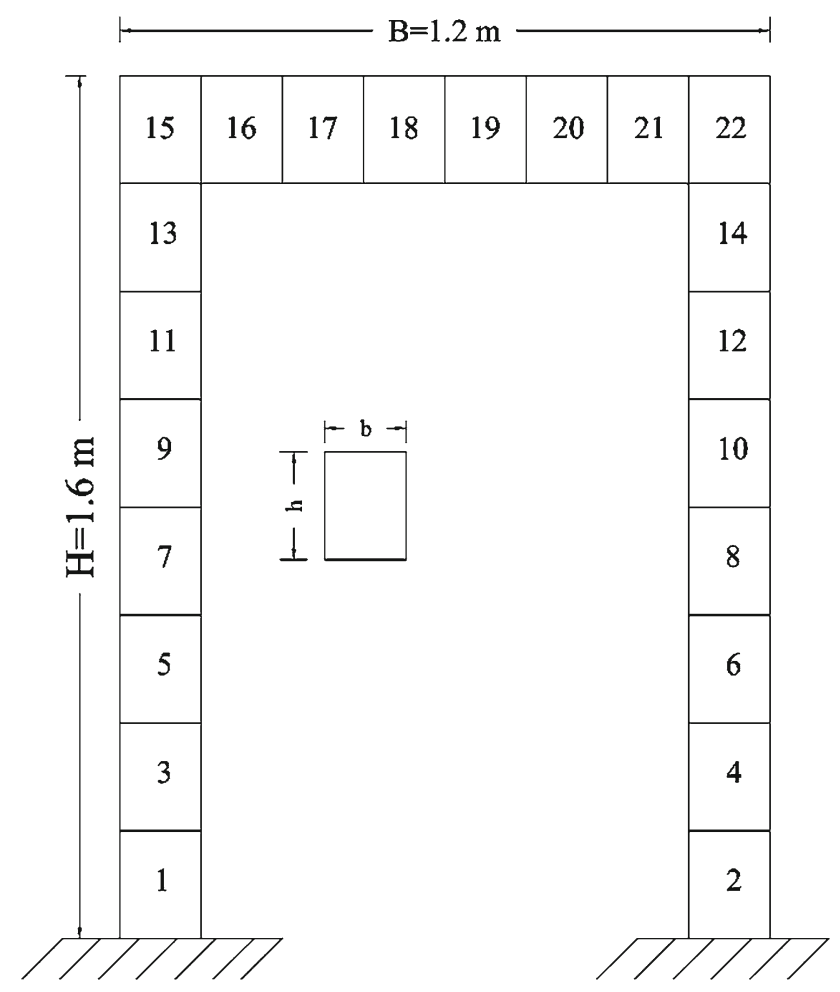

Fig. 10 Portal plane frame considered to validate the proposed damage detection methods

\subsection{A portal frame}

In this section, a one-story portal frame is utilized to investigate the effectiveness of proposed damage detection methods as shown in Fig. 10. Corresponding model of the frame is constructed according to fundamental concepts of finite element method by using two-node beam elements. Hence, number of nodes and elements of the portal frame are 21 and 22, respectively. Subsequently, modal analysis is carried out to identify the structure's modal parameters. It is assumed that all portal frame's members have rectangular cross sectional of $h=0.2 \mathrm{~m}$ height and $\mathrm{b}=0.15 \mathrm{~m}$ width. Furthermore, the frame's length and height are $\mathrm{B}=1.2 \mathrm{~m}$ and $\mathrm{H}=1.6 \mathrm{~m}$, respectively. Properties considered for the frame's material are Young Modulus (E) $2.5 \times 10^{10} \mathrm{~N} / \mathrm{m}^{2}$ and density 2,500 $\mathrm{kg} / \mathrm{m}^{3}$.

Here, four damage cases are considered to explore capabilities of proposed methods for detecting occurred damages in the portal frame. Hence, in the first damage case, stiffness of element 3 is decreased by $20 \%$. This case is near to columnfoundation joint that is a significant part of columns, particularly in the reinforced concrete structures. In the second damage case, stiffness of elements 6,10 and 14 are reduced by 15,20 and $20 \%$, respectively. Damages in three different parts of frame's column comprising of two mid-span points and a point close to beam-column joint are assessed in this 


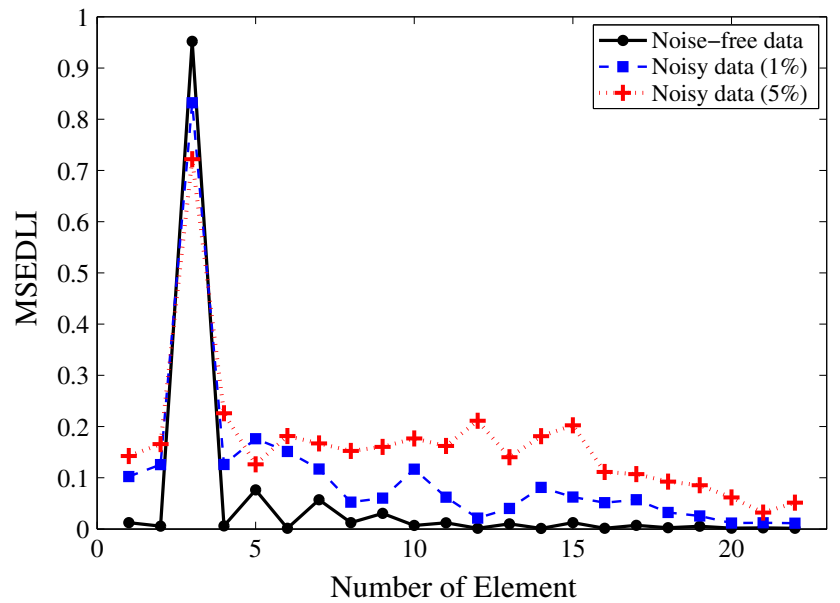

Fig. 11 Damage localization in the portal frame by MSEDLI (damage case 1)

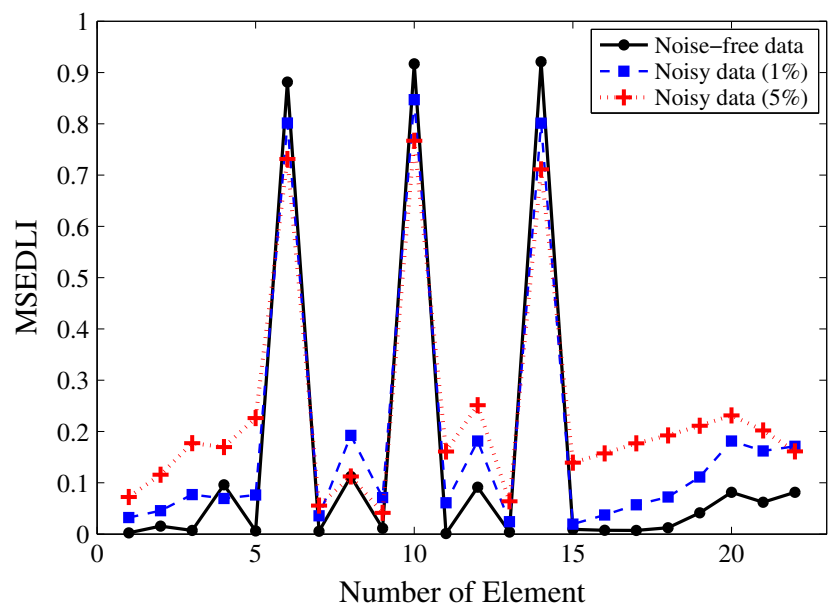

Fig. 12 Damage localization in the portal frame by MSEDLI (damage case 2)

damage case. In the third damage case, importance of the beam-column connection is evaluated. Thus, stiffness of element 15 and 22 are decreased by $30 \%$. Finally, in the damage case number four, stiffness of element 18 and 19 reduced by 10 and $15 \%$, respectively. Damage occurred at the mid-span of a portal frame's beam is assessed in this case.

Similarly to previous section, introduced damages can be localized by correlation of modal strain energy. It should be mentioned that only the frame's first three modes of vibration are utilized to create incomplete condition for modal identification. Subsequently, the both undamaged and damaged modal parameters are contaminated by two types of random noises containing 1 and $5 \%$, respectively. Thus, Figs. 11, 12, 13 and 14 illustrate locations of imposed damages in different damage cases.

It is clear from the above diagrams that damage sites are precisely detected when both incomplete and contaminated

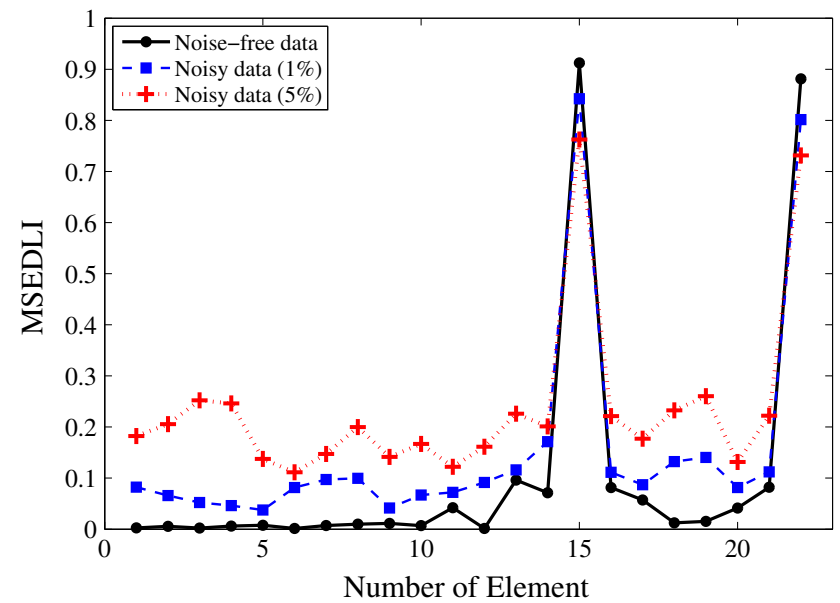

Fig. 13 Damage localization in the portal frame by MSEDLI (damage case 3)

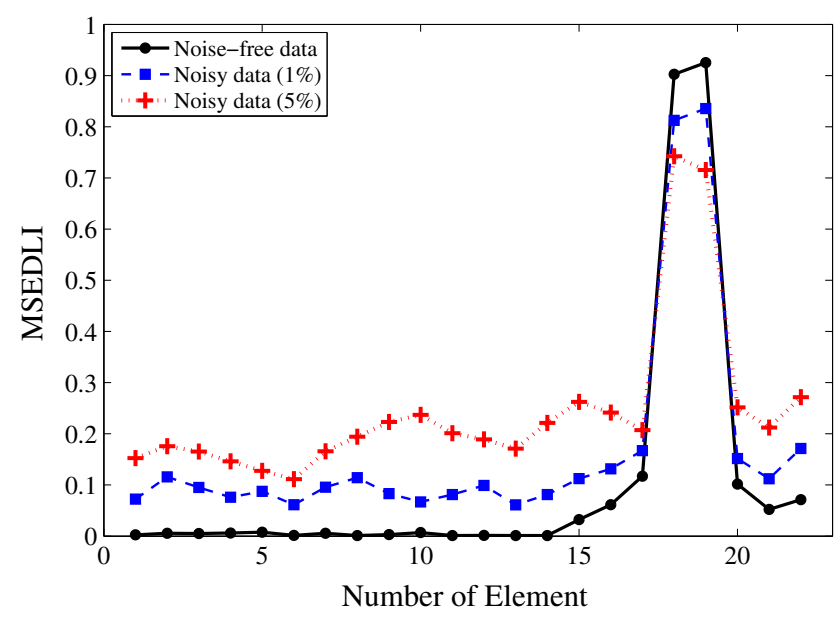

Fig. 14 Damage localization in the portal frame by MSEDLI (damage case 4)

modal data are present. Hence, Figs. 11, 12, 13 and 14 illustrate that the highest peaks (points) of MSEDLI diagrams detect the damage sites precisely. Moreover, the computational errors in the damaged elements are approximately less than 8,12 and $16 \%$ for all types of noisy data including noisefree data, 1 and $5 \%$ random noises, respectively. Although, the noisy data can corrupt the MSEDLI diagrams, the results of damage localization have appropriately been detected by correlation of modal strain energy. It should be noticed that the modal strain energy is calculated by only the first three portal frame's modes of vibration. Thus, correct estimation of the initial properties of the structure particularly the stiffness matrix may be the reason for theses accurate results. In other words, damage localization process using modal strain energy directly depends on precise determination of structure's stiffness matrix. Therefore, it is of great significance to provide proper initial information regarding physical prop- 


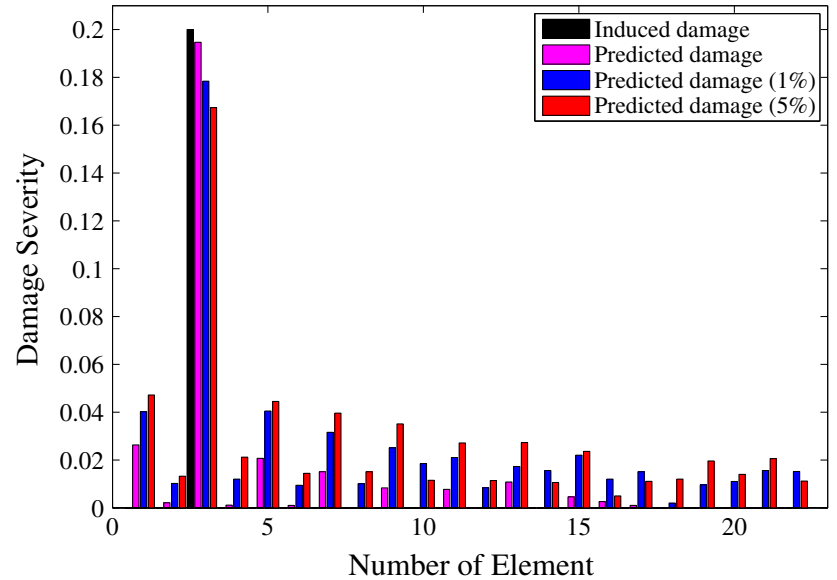

Fig. 15 Damage quantification in the portal frame (damage case 1)

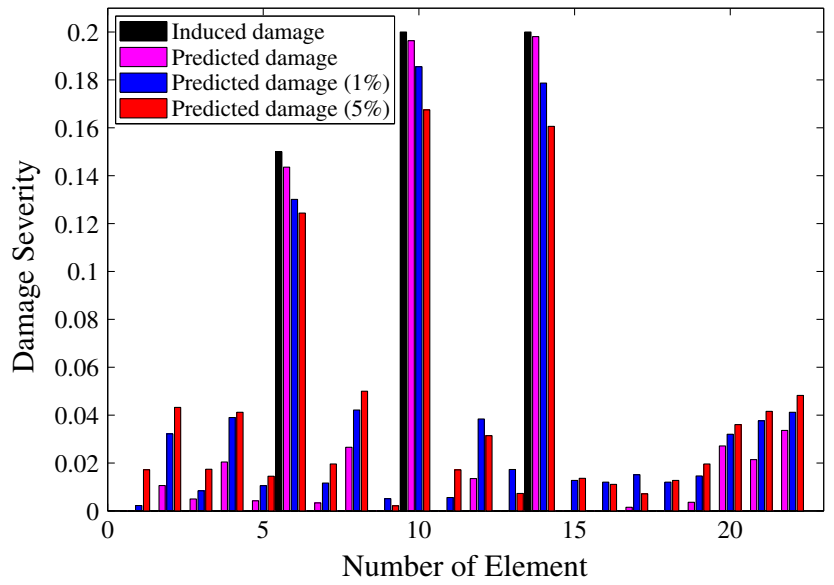

Fig. 16 Damage quantification in the portal frame (damage case 2)

erties of the structure for detecting its future damages. Furthermore, in practice, estimation of stiffness matrix is generally carried out by measured modal parameters. Hence, provided that real modal data of the structure are identified, its mass and stiffness matrices will be estimated by an acceptable precision [36-39]. After damage localization process, damage quantities are determined using improved sensitivity matrix of undamaged portal frame and Tikhonov regularization approach. As a result, Figs. 15, 16, 17 and 18 indicate damage severities induced in the frame in different damage cases as well as three levels of noise effects.

Comparing the imposed damage cases and predicted damages, it is clear that the proposed method for damage quantification has impressive performance in estimating local structural damage severities. Indeed, negligible amounts of error in predicting damages indicate effectiveness of proposed method. Moreover, it can be observed that damages in the portal frame are precisely detected particularly in significant members like column, beam and connections. However, it should be mentioned that obtained damage in the

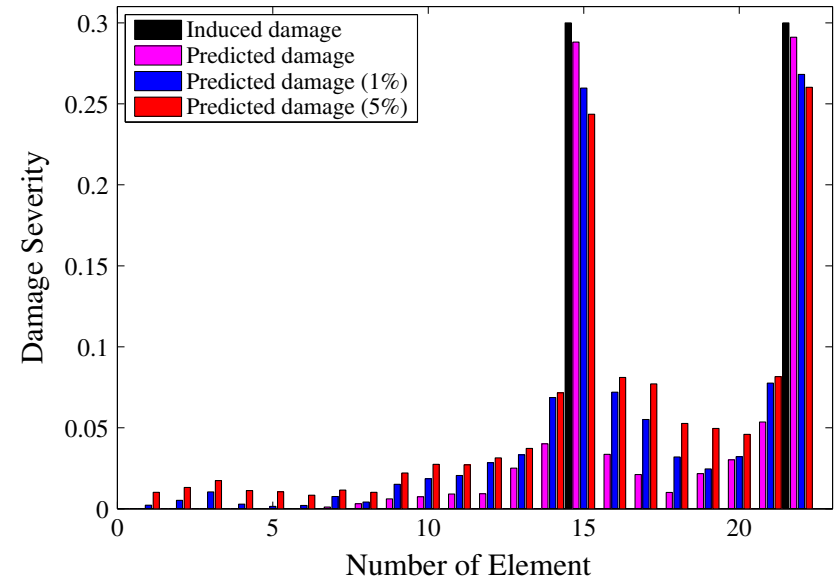

Fig. 17 Damage quantification in the portal frame (damage case 3)

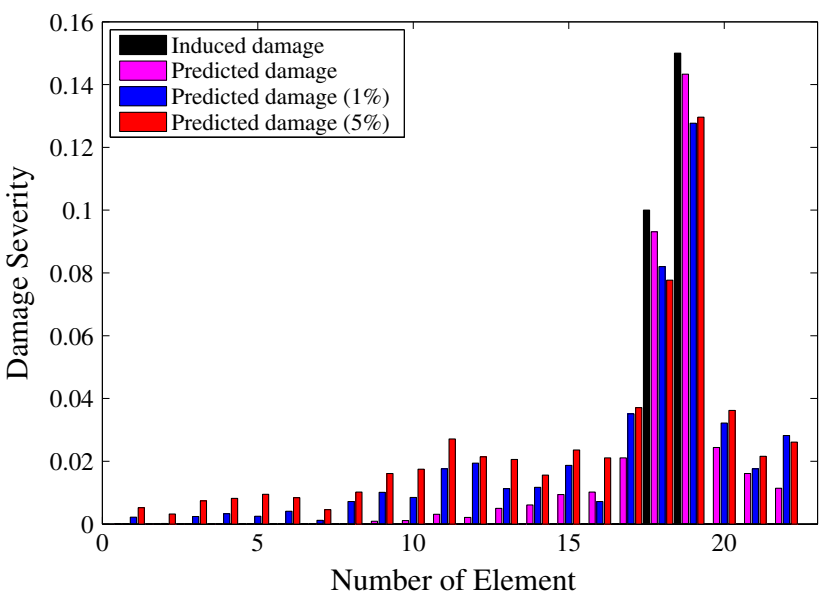

Fig. 18 Damage quantification in the portal frame (damage case 4)

beam-column joints has more influence on dynamic behavior of the structure in comparison with other members of the frame. Owing to the fact that stability of columns is of greatest importance, correct estimation of damages in these members is an indication of proposed method's effectiveness.

\section{Conclusion}

In this paper, location and severity of damage in structural systems is investigated by improved vibrational-based techniques. Accordingly, a correlation between modal strain energy of undamaged and damaged structures is presented to precisely identify structural damage locations in the cases of incomplete and contaminated modal data. This process obviates some of the weaknesses in direct changes of modal strain energy which were proposed by other researchers. For damage quantification, an improved sensitivity of modal strain energy is generated to be applied in damage equation as penalty function. This function is formulated by truncated 
Taylor series. Subsequently, a capable minimizing process for damage equation is used instead of employing current inverse problem techniques. Hence, Tikhonov regularization method is utilized and an objective function related to damage equation is minimized to determine damage severities. It is shown that the proposed method is preferable and can provide more reliable and accurate results in comparison with other classical techniques. In order to assess the performance of proposed methods, two practical systems including a planner truss and a portal frame are considered to detect the induced damage cases. The numerical results demonstrate that correlation of modal strain energy and applying the Tikhonov regularization can provide an efficient tool for proper identification of multiple damages in structural systems when incomplete modes are present and modal data are contaminated by random noise.

\section{References}

1. Doebling SW, Farrar CR, Prime MB and Shevitz DW (1996) "Damage identification and health monitoring of structural andmechanical systems from changes in their vibration characteristics: a literature review", Research Rep. No. LA-13070-MS, ESA-EA, Los Alamos National Laboratory NM, USA, 1996.

2. Stubbs N, Broome TH, Osegueda R (1990) Nondestructive construction error detection in large space structures. AIAA J 28:146152

3. Salawu OS (1997) Detection of structural damage through changes in frequency: a review. Eng Struct 19:718-723

4. Yan YJ, Cheng L, Wu ZY, Yam LH (2007) Development in vibration-based structural damage detection technique. Mech Syst Signal Process 21:2198-2211

5. Yan A, Golinval JC (2005) Structural damage localization by combining flexibility and stiffness methods. Eng Struct 27:1752-1761

6. Yang QW, Sun BX (2011) Structural damage identification based on best achievable flexibility change. Appl Math Model 35:52175224

7. Kim J-T, Ryu Y-S, Cho H-M, Stubbs N (2003) Damage identification in beam-type structures: frequency-based method versus mode-shape-based method. Eng Struct 25:57-67

8. Esfandiari A, Bakhtiari-Nejad F, Rahai A (2013) Theoretical and experimental structural damage diagnosis method using natural frequencies through an improved sensitivity equation. Int J Mech Sci 70:79-89

9. Yan W-J, Ren W-X, Huang T-L (2012) Statistic structural damage detection based on the closed-form of element modal strain energy sensitivity. Mech Syst Signal Process 28:183-194

10. Hu H, Wu C, Lu W-J (2011) Damage detection of circular hollow cylinder using modal strain energy and scanning damage index methods. Comput Struct 89:149-160

11. Shi Z, Law S, Zhang L (2000) Damage localization by directly using incomplete mode shapes. J Eng Mech 126:656-660

12. Seyedpoor SM (2012) A two stage method for structural damage detection using a modal strain energy based index and particle swarm optimization. Int J Non-Linear Mech 47: $1-8$

13. Shi ZY, Law SS (1998) Structural damage localization from modal strain energy change. J Sound Vib 215:825-844

14. Ewins DJ (2000) Modal testing: theory and practice and application, 2nd edn. Wiley, Chichester
15. Li L, Hu Y, Wang X (2013) Numerical methods for evaluating the sensitivity of element modal strain energy. Finite Elem Anal Des 64:13-23

16. Yan WJ, Ren WX (2011) A direct algebraic method to calculate the sensitivity of element modal strain energy. Int J Numer Methods 27:694-710

17. Wang L, Chan THT, Thambiratnam D, Tan A (2010) "Damage detection for truss bridge structures using correlation-based structural modal strain energy", presented at the Proceedings of the 8th International Conference on Short and Medium Span Bridges, Niagara Falls, Ontario, Canada

18. Koh BH, Dyke SJ (2007) Structural health monitoring for flexible bridge structures using correlation and sensitivity of modal data. Comput Struct 85:117-130

19. Messina A, Williams E, Contursi T (1998) Structural damage detection by a sensitivity and statistical-based method. J Sound Vib 216:791-808

20. Hansen PC (1998) Rank-deficient and discrete ill-posed problems: numerical aspects of linear inversion. Society for Industrial and Applied Mathematics, Philadelphia

21. Ambrogio WD, Fregolent A (1998) On the use of consistent and significant information to reduce ill-conditioning in dynamic model updating. Mech Syst Signal Process 22(1):203-222

22. Ren WX (2005) A singular value decomposition based truncation algorithms in solving the structural damage equation. Acta Mech Solida Sinica 18:181-188

23. Tikhonov AN (1995) Numerical methods for the solution of Illposed problems. Kluwer Academic Publishers, Boston

24. Ahmadian H, Mottershead JE, Friswell MI (1998) Regularisation methods for finite element model updating. Mech Syst Signal Process 12:47-64

25. Fiswell MI, Mottershead JE, Ahmadian H (2001) Finite-element model updating using experimental data: parameterization and regularization. Philos Trans R Soc Lond, Ser A-Math Phys Eng Sci 359:169-186

26. Hua XG, Ni YQ, Ko JM (2009) Adaptive regularization parameter optimization in output-error-based finite element model updating. Mech Syst Signal Process 23:563-579

27. Ziaei-Rad S, Imregun M (1999) On the use of regularisation techniques for finite element model updating. Inverse Probl Eng 7:471503

28. Hansen PC (1992) Analysis of discrete ill-posed problems by means of the L-curve. SIAM Rev 34:561-580

29. Calvetti D, Reichel L, Zhang Q (1999) Iterative solution methods for large linear discrete ill-posed problems. Appl Comput Control, Signals Circuits 1:313-367

30. Calvetti D, Morigi S, Reichel L, Sgallari F (2000) Tikhonov regularization and the L-curve for large, discrete ill-posed problems. J Comput Appl Math 123:423-446

31. Wahba G (1977) Practical approximate solutions to linear operator equations when the data are noisy. SIAM J Numer Anal 14:651667

32. Golub GH, Heath M, Wahba G (1979) Generalized cross-validation as a method for choosing a good ridge parameter. Technometrics $21: 215-223$

33. Lukas MA (1993) Asymptotic optimality of generalized crossvalidation for choosing the regularization parameter. Numer Math 66:41-66

34. Rahai A, Bakhtiari-Nejad F, Esfandiari A (2007) Damage assessment of structure using incomplete measured mode shapes. Struct Control Health Monit 14:808-829

35. Rao SS (2005) The finite element method in engineering. Butterworth-Heinemann publications, Amsterdam, Boston, Heidelberg, London, New York, Oxford Paris, San Diego, San Francisco, Singapore, Sydney, Tokyo 
36. Chakraverty S (2004) Identification of structural parameters of multistorey shear buildings from modal data. Earthq Eng Struct Dyn 34:543-554

37. Kabe A (1985) Stiffness adjustment using mode data. AIAA J 23:1431-1436

38. Lee ET, Eun HC (2009) Update of corrected stiffness and mass matrices based on measured dynamic modal data. Appl Math Model 33:2274-2281
39. Omrani R, Hudson RE, Taciroglu E (2012) Story-by-story estimation of the stiffness parameters of laterally-torsionally coupled buildings using forced or ambient vibration data: I. Formulation and verification. Earthq Eng Struct Dyn 41:1609-1634 